\title{
Improving polarized neutron imaging for visualization of the Meissner effect in superconductors
}

Cite as: Rev. Sci. Instrum. 90, 033705 (2019); https://doi.org/10.1063/1.5053690

Submitted: 24 August 2018 . Accepted: 27 February 2019 . Published Online: 18 March 2019

T. Wang, C. Y. Jiang (D, H. Z. Bilheux (D), I. Dhiman, J. C. Bilheux, L. Crow (D), L. McDonald, L. Robertson, N. Kardjilov, R. Pynn (D), and X. Tong (iD)

\section{ARTICLES YOU MAY BE INTERESTED IN}

Setup for polarized neutron imaging using in situ ${ }^{3} \mathrm{He}$ cells at the Oak Ridge National Laboratory High Flux Isotope Reactor CG-1D beamline

Review of Scientific Instruments 88, 095103 (2017); https://doi.org/10.1063/1.5001525

Determine turntable coordinate system considering its non-orthogonality

Review of Scientific Instruments 90, 033704 (2019); https://doi.org/10.1063/1.5084799

Measurement of the injecting time of picosecond laser in indirect-drive integrated fast ignition experiments using an x-ray streak camera

Review of Scientific Instruments 90, 033504 (2019); https://doi.org/10.1063/1.5050039

\begin{tabular}{|c|c|c|c|c|}
\hline $\begin{array}{c}\text { Nanopositioning } \\
\text { Systems }\end{array}$ & $\begin{array}{c}\text { Modular } \\
\text { Motion Control }\end{array}$ & $\begin{array}{c}\text { AFM and NSOM } \\
\text { Instruments }\end{array}$ \\
\hline MAD CITY LABS INC. \\
Wwwadcitylabs.com
\end{tabular}

Rev. Sci. Instrum. 90, 033705 (2019); https://doi.org/10.1063/1.5053690 


\title{
Improving polarized neutron imaging for visualization of the Meissner effect in superconductors
}

\author{
Cite as: Rev. Sci. Instrum. 90, 033705 (2019); doi: 10.1063/1.5053690 \\ Submitted: 24 August 2018 - Accepted: 27 February 2019 • \\ Published Online: 18 March 2019
}

T. Wang, ' C. Y. Jiang, ${ }^{1}$ (D) H. Z. Bilheux, (D) I. Dhiman, ' J. C. Bilheux, L. Crow, 'iD L. McDonald, L. Robertson, N. Kardjilov, ${ }^{2}$ R. Pynn, ${ }^{1,3}$ (D) and X. Tong ${ }^{1,4,5, a)}$ (D)

\begin{abstract}
AFFILIATIONS
${ }^{1}$ Neutron Science Directorate, Oak Ridge National Laboratory, Oak Ridge, Tennessee 37831, USA

${ }^{2}$ Institute Applied materials, Helmholtz Zentrum Berlin, Berlin, Germany

${ }^{3}$ Department of Physics, Indiana University Bloomington, Bloomington, Indiana 47405, USA

${ }^{4}$ Institute of High Energy Physics, Chinese Academy of Sciences (CAS), Beijing 100049, China

${ }^{5}$ Dongguan Neutron Science Center, Dongguan 523803, China
\end{abstract}

a) Author to whom correspondence should be addressed: tongx@ihep.ac.cn.

\begin{abstract}
The polarized neutron imaging technique provides a non-invasive method of characterizing localized magnetic fields inside superconductors. However, complete understanding of the magnetic field distribution has yet to be realized experimentally due to the complexity of the interaction between neutron polarization and magnetic field. In this article, we show that a well-defined and controlled magnetic field through the neutron path contributes to simplify the data analysis and makes future quantitative polarized neutron imaging possible. This is demonstrated in a set of experiments that visualize the magnetic field distribution inside and around the superconductors. The experimental results demonstrate that proper guide field setup allows the visualization of the magnetic field expulsion at the surface of the superconductor in the zero-field cooling condition, as well as the magnetic field trapped inside the superconductor under field cooling condition.
\end{abstract}

Published under license by AIP Publishing. https://doi.org/10.1063/1.5053690

\section{INTRODUCTION}

The distribution of magnetic fields inside a superconductor is often interesting to researchers, but is difficult to be measured accurately. Conventional characterization methods such as Small Angle Neutron Scattering ${ }^{\top}$ (SANS) or Scanning Tunneling Microscope $(\mathrm{STM})^{2}$ either measure the magnetization of the whole sample without giving localized magnetization distribution information or only measure the magnetization distribution on the sample surface. Polarized Neutron Imaging (PNI), first demonstrated in $2008,3,4$ provides a way of probing magnetic structures inside samples. This is achieved through recording the polarization shift of the neutron due to its Larmor precession within a magnetic field. The final neutron polarization is an integral of magnetic-field along the neutron path just as a conventional transmission radiograph is an integral of neutron attenuation along the path. The neutron's non-destructive nature along with its deep penetration makes PNI a unique technique for studying magnetic fields inside samples. ${ }^{5,6}$ This technique has been implemented at several neutron imaging beamlines. 7,8

The behavior of the neutron polarization in a magnetic field is described by the Bloch equation,

$$
\frac{d \vec{P}}{d t}=-\gamma \vec{P} \times \vec{B}
$$

where $\boldsymbol{P}$ is the neutron polarization, $\boldsymbol{B}$ is the external magnetic field, and $Y$ is the neutron gyromagnetic ratio. The evolution of the neutron polarization defined by Eq. (1) can be summarized as neutron polarization vector precessing around the external magnetic field while following the field direction change. This process can be visualized as a "rolling-cone" construction, ${ }^{9}$ as shown in Fig. 1. The opening angle of the cone, $2 \phi$, is given by the ratio of the rate of 


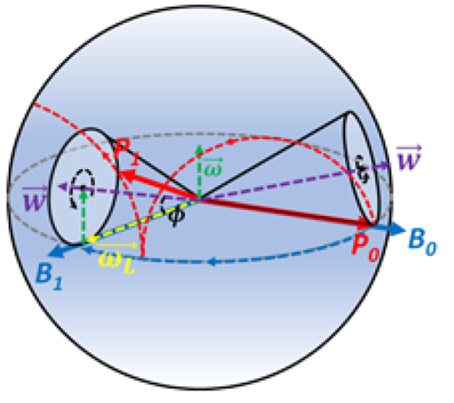

$\longrightarrow P_{0}$ Initial neutron polarization

$\longrightarrow P_{1}$ Final neutron polarization

$\longrightarrow B_{0}$ Initial magnetic field

$\longrightarrow B_{1}$ Final magnetic field

$\longrightarrow \omega$ Magnetic field rotation angular vector

Larmor precession angular vector

$w$ Combined angular vector
FIG. 1. Rolling-cone construction of neutron polarization $P$ revolving around a varying magnetic field $\mathbf{B}$. The neutron polarization vector, $\mathbf{P}$, lies on the cone and initially coincides with the magnetic field direction, which is marked on the equatorial plane of a sphere as shown. As the tip of the field vector moves around the equator, the cone rolls on the equatorial plane with its tip fixed at the center of the sphere so that it always contacts that plane along the line of the $\mathbf{B}$ vector. Meanwhile the neutron polarization follows the $\mathbf{P}$ vector marked on the cone. rotation of the $\boldsymbol{B}$ field, $\omega$, and the Larmor frequency $\omega_{L}=\gamma B$ as

$$
\operatorname{Tan}[\phi]=\omega / \omega_{L}
$$

If the field rotates slowly relative to the Larmor frequency (adiabatic case), the opening angle $\phi$ is small, and the neutron polarization follows the magnetic field as its direction changes. While at the other extreme, when the field suddenly changes direction (extreme nonadiabatic case), the polarization simply precesses around the new field direction at the Larmor frequency. Beyond these two cases, the neutron polarization revolves around a time-varying magnetic field in a complicated manner, which can be solved based on Eq. (1) using an algorithm developed by Seeger and Daemen. ${ }^{10}$

When utilizing PNI to probe unknown magnetic fields, it is clear from the above that the information obtained from a PNI experiment is often difficult to interpret due to the complex polarization vector motion. ${ }^{11}$ To extract useful information, it is necessary to experimentally maintain the neutron polarization in an adiabatic or extreme non-adiabatic condition outside the sample magnetic field region. Thus, the changes in neutron polarization due to the sample can be isolated and considered as a series of small regions through which the neutron passes. In each region where the magnitude of the magnetization is approximately constant, the change in direction of the neutron polarization is simply described by multiplying $\boldsymbol{P}$ by a rotation matrix defined by three Euler angles whose values can be calculated from the Bloch equation. Accordingly, the final polarization is the result of multiplying these matrices together along the neutron path. In this paper, we focus on the experimental setup used for PNI measurement of superconductors and explore the ways in which the neutron polarization can be controlled. The experimental results in the paper demonstrate that the geometry and the boundary conditions of the superconductor are essential to establishing neutron polarization control and the quantitative reconstruction of the sample magnetization.

The example superconductor we examine in this article is YBCO, a type-II superconducting material. This material exhibits a Meissner effect that, under Zero-Field-Cooling (ZFC), expels the external magnetic field and forbids a magnetic field orthogonal to its surface. ${ }^{12,13}$ In this case, as shown in Fig. 2(a), the magnetic guide fields can be applied parallel to the surfaces, and the neutron polarization can be guided adiabatically following the magnetic field. However, as shown in Fig. 2(b), neutron polarization orthogonal to the superconductor surface is not achievable through adiabatically following the magnetic field because the Meissner effect constrains the field to be parallel to the sample surface. The Meissner effect also causes the superconductor to trap the external magnetic field during Field-Cooling (FC) across its critical temperature. Two FC cases are investigated, as shown in Figs. 2(c) and 2(d). In the case of Fig. 2(c), where the trapped field is parallel to the surface through which neutrons pass, the neutron polarization experiences a non-adiabatic transition at the sample surface and simply precesses around the magnetic field inside the sample. The total precession angle, $\varphi_{L}$, within the sample is simply related to the line integral of the sample magnetization along the neutron trajectory as

$$
\varphi_{L}=\gamma B t=\frac{\gamma m}{h} \lambda \cdot \int_{p a t h} B(l) d l,
$$
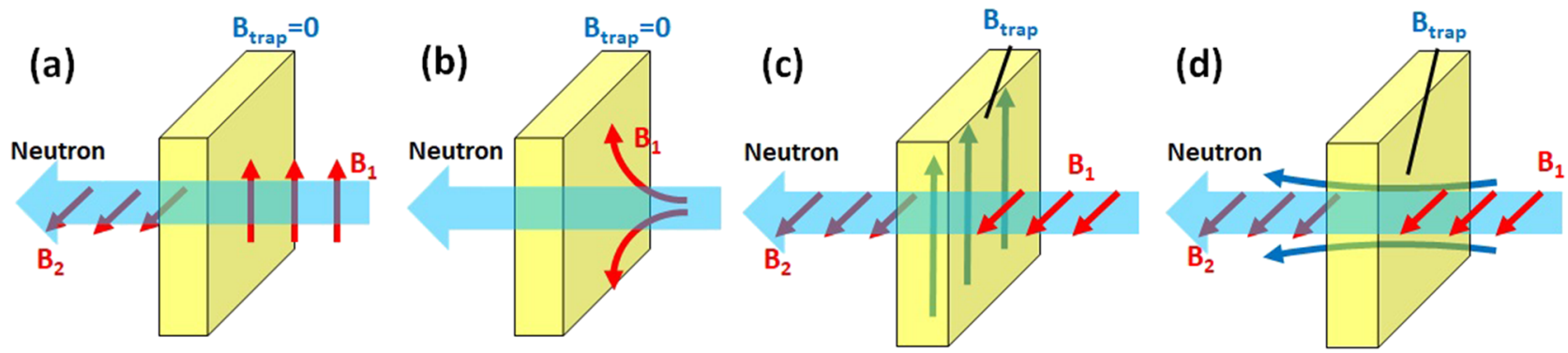

FIG. 2. Guide field configuration around superconductors investigated in this article, where $B_{\text {trap }}$ is the magnetic field inside the superconductor and $B_{1}, B_{2}$ are external guide fields on opposite sides of the sample. In (a) and (b), the sample is cooled in ZFC condition so that there is no magnetic field inside the sample. The external magnetic fields are applied parallel and perpendicular to the sample surface in (a) and (b), respectively. In (c) and (d), the sample is treated in the FC condition and therefore contains a trapped internal magnetic field parallel or perpendicular to its surface. The external magnetic fields in (c) and (d) are both applied parallel to the sample surface. 
where $m$ is the mass of neutron, $h$ is Planck's constant, $\lambda$ is the neutron wavelength, and $d l$ is the infinitesimal segment along the neutron path. For the case of Fig. 2(d) where the trapped field is orthogonal to the surface, the trapped field overlaps with external magnetic field and thus creates a complicated surface condition.

\section{INSTRUMENTATION SETUP}

The PNI experiments were performed at the imaging beamline (CG-1D) at the High Flux Isotope Reactor (HFIR) at the Oak Ridge National Laboratory. The beamline provides a steady beam of single wavelength $(\lambda=2.53 \AA$ ) neutrons polarized by supermirrors and an in situ Spin Exchange Optically Pumped (SEOP) polarized ${ }^{3} \mathrm{He}$ system. ${ }^{14}$ A similar setup can be found in an earlier publication. ${ }^{15}$ The schematics and pictures of the experiment setup are shown in Fig. 3. We use a set of rotatable electromagnets [Fig. 3(b)] to provide a well-controlled magnetic field parallel to the sample surface. These electromagnets are equipped with an angular scale so that the direction of the guide field can be read off with $0.5^{\circ}$ angular precision. A set of solenoids is used to generate the magnetic fields perpendicular to the sample surface.

Two types of commercially available YBCO are selected as our superconducting samples. The first sample is a single crystal YBCO thin film, prepared by Ceraco ${ }^{(}$. This thin film sample, with dimensions of $100 \mathrm{~mm} \times 70 \mathrm{~mm}$ and a thickness of $350 \mathrm{~nm}$, is deposited on a $0.5 \mathrm{~mm}$ thick single crystal sapphire. The second sample is a single crystal YBCO sample block, which is provided by Evico $\left.{ }^{(}\right)$and has a dimension of $45 \mathrm{~mm} \times 45 \mathrm{~mm} \times 10 \mathrm{~mm}$ and its c-axis parallel to the neutron beam. Both samples have a critical temperature $T_{c}$ of $\sim 90 \mathrm{~K}$ and are cooled using a two-stage Closed-Cycle-Refrigerator (CCR) (Sumitomo CH-204), capable of reaching a base temperature of $22 \mathrm{~K}$. For the ZFC experiment, the superconducting sample is cooled while enclosed inside a mu-metal enclosure to shield against ambient fields. During the experiment, we use a rectangular shape vacuum tail to maintain the cryogenic cooling while minimizing the distance between the electromagnets and the sample to $2 \mathrm{~cm}$. Both samples are placed inside the vacuum tail with their larger surfaces perpendicular to the neutron beam.

\section{RESULTS AND DISCUSSION}

The data collected from the experiments are the measured intensities of the "spin-up" $\left(I_{+}\right)$and "spin-down" $\left(I_{-}\right)$states of the neutron beam, defined as the neutron spin being parallel or antiparallel to the direction of the neutron polarization analyzer (the SEOP ${ }^{3} \mathrm{He}$ filter). From the measured neutron intensity, we calculate the neutron polarization changes due to the sample, $P_{\text {Sample }}$, as

$$
P_{\text {Sample }}=\frac{I_{+}-I_{-}}{I_{+}+I_{-}} \cdot \frac{1}{A \cdot P},
$$

where $A$ is the ${ }^{3} \mathrm{He}$ filter's analyzing power and $\mathrm{P}$ is the supermirror's polarizing power. The combination of A.P is determined to be $(0.89$ \pm 0.01 ) by measuring the neutron polarization without the sample.

We first explore the setup where the superconducting YBCO film sample is cooled in ZFC condition and then placed between two different magnetic guide fields. As shown in Fig. 4(a), two magnetic guide fields $\boldsymbol{B}_{1}$ and $\boldsymbol{B}_{2}$ are generated by a solenoid and an electromagnet before and after the superconducting YBCO film, respectively. The electromagnets on the back side generate a uniform magnetic field ${ }^{16}$ of $20 \mathrm{G}$, defined by its orientation, up to the superconductor surface. The front solenoid magnetic field diverges and becomes parallel to the superconductor plane at the surface, aligning with the radial direction pointing from the center axis of the solenoid. The field distribution along the neutron flight path around the superconductor is simulated using Magnet ${ }^{\circledR}$, a finite element analysis-based software, as shown in Fig. 4(b). The simulation shows continuous field distribution from the solenoid and the magnets while an abrupt shift occurs at the YBCO film position. The magnetic field distribution from the solenoid at the front surface of the YBCO film is also simulated, as shown in Fig. 4(c). The simulation shows that the magnetic field is parallel to the surface of the superconductor pointing toward or away from the center of the solenoid.

The neutron is first adiabatically guided by the solenoid magnetic field so that their polarization directions align with the diverging magnetic field at the front surface of the superconductor. Crossing the superconducting thin film, neutrons undergo an extreme non-adiabatic field transition from $\boldsymbol{B}_{1}$ to $\boldsymbol{B}_{2}$. If we define $\theta$ as the

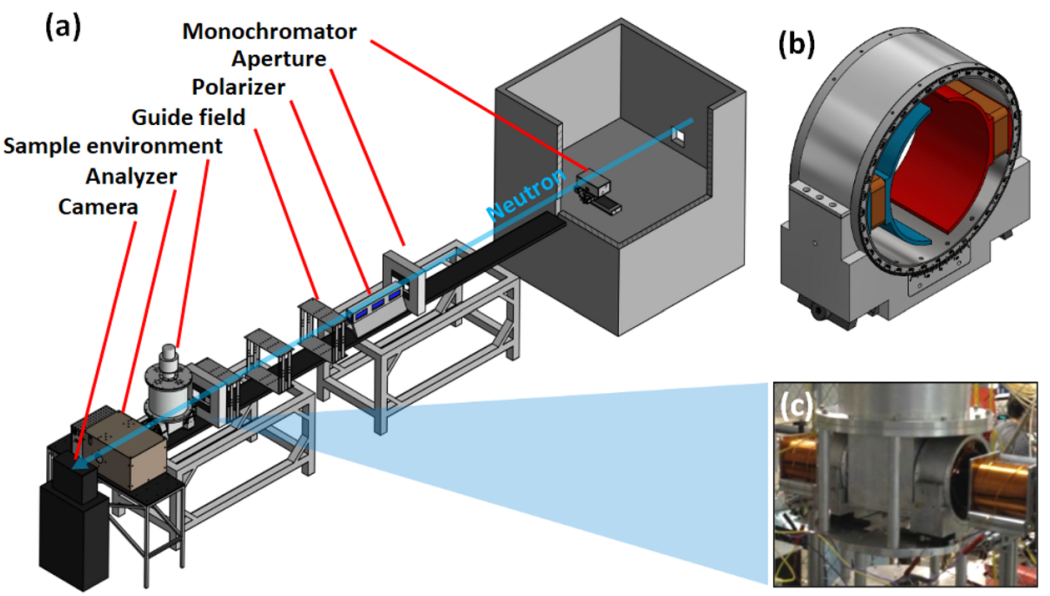

FIG. 3. Layout of the experimental setup at the CG-1D beamline at HFIR, ORNL: (a) Schematics of the experimental setup; (b) schematics of the rotatable electromagnets used as guide fields around the sample; (c) photo of the magnetic field setup around the sample. 

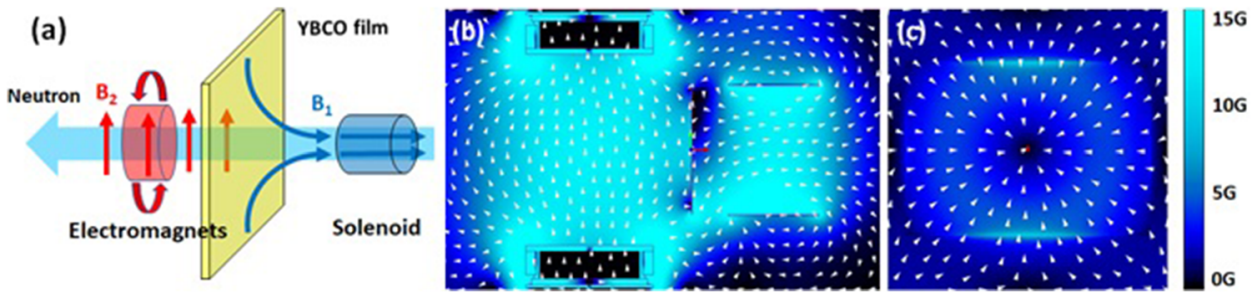

FIG. 4. (a) Relative position of the external guide fields, superconductor, and magnetic field. (b) Finite element simulation of the magnetic field distribution around the superconductor (side view); the film position is marked by a white arrow, and the neutron flight path is labeled by the black dashed arrow. (c) Simulation of the magnetic field distribution from the solenoid at the surface of the superconductor. The field magnitude is indicated by the colors, while the direction is labeled by white arrows.
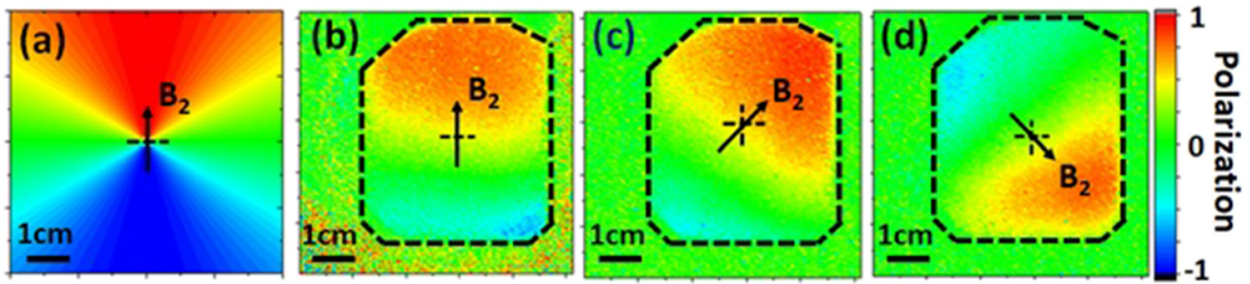

FIG. 5. Comparison of simulated neutron polarization and PNI measurement results: (a) neutron polarization with $B_{2}$ vertically up calculated from Eq. (5); (b) PNI measurement result of neutron polarization with $B_{2}$ vertically up; (c) measured neutron polarization with electromagnets rotated $45^{\circ}$ clockwise from the vertical direction; (d) measured neutron polarization with electromagnets rotated $135^{\circ}$ clockwise from the vertical direction. The effective sample area is labeled by the black dashed line, and the solenoid center position is labeled by the black dashed cross.

angle between $\boldsymbol{B}_{1}$ and $\boldsymbol{B}_{2}$, the measured neutron polarization after the superconductor is given by

$$
P_{\text {sample }}=\operatorname{Cos}(\theta)=\frac{\vec{r} \cdot \vec{z}}{|\vec{r}|},
$$

where $\mathbf{r}$ is the position vector from the center of the solenoid and $\mathbf{z}$ is the direction of $\boldsymbol{B}_{2}$. In the ideal case where all transitions in solenoid field $\boldsymbol{B}_{1}$ are adiabatic, the polarization distribution as a function of position is calculated from Eq. (5) and demonstrated in Fig. 5(a). The corresponding measured PNI result with the same magnetic field configuration is shown in Fig. 5(b).

Comparison between the calculated [Fig. 5(a)] and measured results [Fig. 5(b)] confirms that the field distribution predicted by Eq. (5) is qualitatively correct. We further examine the correlation between the $B_{2}$ field direction and the neutron polarization distribution from Eq. (5) by rotating the electromagnets $45^{\circ}$ and $135^{\circ}$ clockwise from the vertically upward direction. The measured results in Figs. 5(c) and 5(d) show that the imaging pattern rotation is consistent with the rotation of the electromagnet.

To visualize the field trapped inside superconductors in the FC condition, we first examine the case where the trapped field is parallel to the superconductor surface. The trapped field is generated by placing electromagnets symmetrically on each side of a single YBCO block with the generated magnetic field vertically upward, ${ }^{16}$ shown in Fig. 6(a). By controlling the current through the electromagnets, we selectively apply $7.5 \mathrm{G}$ and $15 \mathrm{G}$ at the center of the sample position. The YBCO block sample is then cooled down through $T_{c}$ within this field. After cooling, we rotate both electromagnets in the horizontal direction, as shown in Fig. 6(b).

The neutron polarization experiences non-adiabatic transitions at the front and back surfaces, where the direction of the magnetic field changes by $90^{\circ}$ ( $\pi / 2$ flip). As a result of the first flip, the neutron polarization precesses around the trapped magnetic field inside the sample where the amount of the precession can be calculated from Eq. (2). The measured polarization is the cosine of the precession angle, given by

$$
P=\operatorname{Cos}\left(\frac{\gamma m}{h} \lambda \cdot B_{\text {trap }} \cdot l\right),
$$

where $l$ is the thickness of the YBCO block $(1 \mathrm{~cm})$ and $B_{\text {trap }}$ is the magnitude of the trapped magnetic field. We compare the result of the two different trapped field magnitudes along with zero trapped field results, as shown in Fig. 7.

All results demonstrate a shift of neutron polarization across the sample, although the non-zero trapped field result shows deviation at the sample boundary. We attribute these deviations to the non-uniformity of the electromagnets' guide fields at the edge of
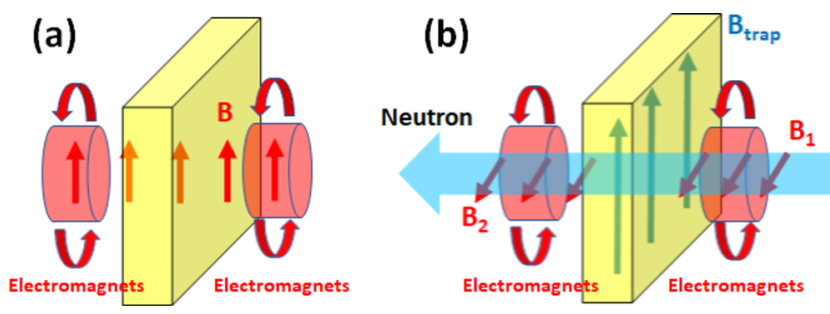

FIG. 6. Experimental setup with a trapped field parallel to the surface the neutrons pass through: (a) relative position of the external guide fields, superconductor, and magnetic field during field trapping and (b) relative position of the external guide fields, superconductor, and magnetic field during measurement. 

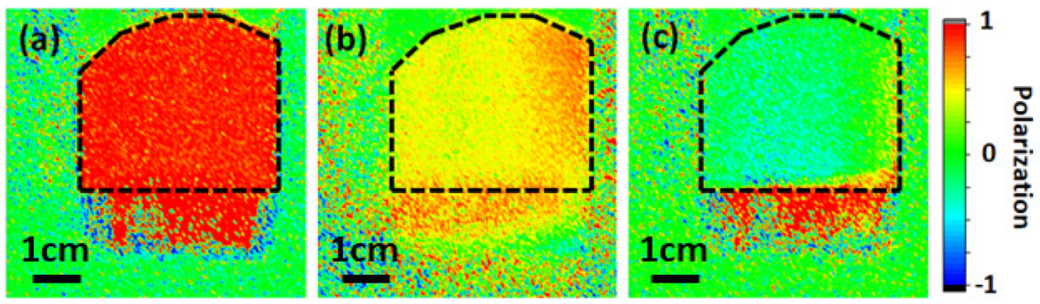

FIG. 7. PNI measurement results of the magnetic field inside superconductors using the setup described in Fig. 6. The sample area is labeled by a black dashed line: (a) zero trapped field, (b) FC trapped field of $7.5 \mathrm{G}$ parallel to the YBCO block surface, and (c) FC trapped field of $15 \mathrm{G}$ parallel to the YBCO block surface.

the sample. To determine the trapped field magnitude, we calculate the neutron polarization shift due to the sample by average across the sample area. The average polarizations are $1.00 \pm 0.01,0.61$ \pm 0.01 , and $-0.20 \pm 0.01$ for the results shown in Figs. $7(\mathrm{a})-7(\mathrm{c})$, respectively. Calculated from Eq. (6), these neutron polarizations correspond to a trapped field of $0.5 \mathrm{G} \pm 1.5 \mathrm{G}, 7.8 \mathrm{G} \pm 0.1 \mathrm{G}$, and $15.1 \mathrm{G} \pm 0.1 \mathrm{G}$. The applied magnetic field and measured trapped field from neutron polarization are close, although a small disparity exists due to slight misalignment between the two electromagnets and background noise.

Lastly, we explore the situation where the FC trapped field is orthogonal to the surface that the neutron passes through. This configuration is first explored by placing a small permanent magnet in front of the YBCO block while cooling through $\mathrm{T}_{\mathrm{c}}$, as shown in Fig. 8(a). The permanent magnet is placed $2 \mathrm{~cm}$ upstream from the YBCO block, generating a $200 \mathrm{G}$ magnetic field on its surface. After cooling, the permanent magnet is removed, and electromagnets are installed to provide guide fields along the vertical [Fig. 8(b)] and horizontal [Fig. 8(c)] directions.

The trapped magnetic field extends beyond the sample boundary and superposes with the external guide field at the surface. This leads to a neutron polarization that varies in complicated manner over the superconductor surface. The complexity of the polarization at the superconductor surface is amplified by the precession due to the trapped field and when neutron leaves the superconductor. The PNI results are shown in Figs. 9(a) and 9(b) with the external magnetic fields vertical and horizontal, respectively.

To simplify the spin evolution process, we repeated our measurement with the YBCO block replaced by a YBCO thin film whose thickness $(350 \mathrm{~nm})$ is so small that the neutron precession within the thin film is less than $0.0008^{\circ}$ and therefore negligible in the measurement. Thus, we eliminate the influence of the magnetic field trapped inside the sample and only investigate the surface effect of the superposition of trapped field and external guide field. The results obtained using the same setup as described in Fig. 8 are shown in Figs. 9(c) and 9(d). These results demonstrate that the polarization distribution pattern follows the rotation of the electromagnetic fields, while the center pattern remains largely unchanged. This result suggests that in the center region where the trapped field $(200 \mathrm{G})$ is much stronger than the external guide field $(15 \mathrm{G})$, the neutron polarization follows the field adiabatically throughout its flight path. This adiabatic behavior allows PNI to identify pinned
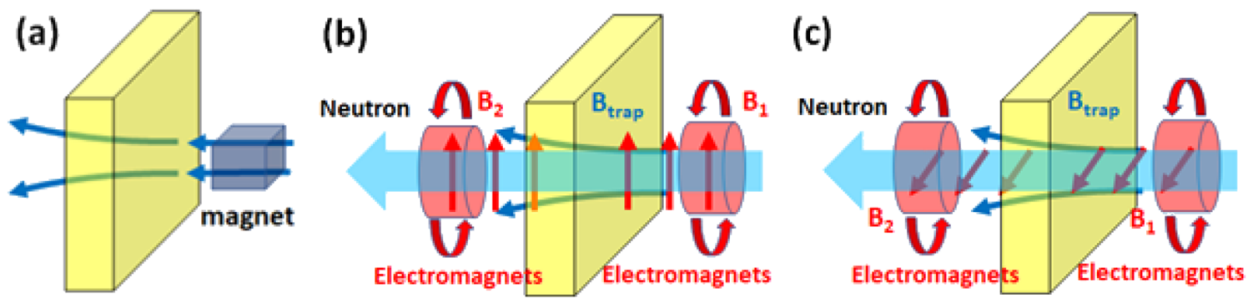

FIG. 8. Experimental setup with a trapped field orthogonal to the surface the neutron passes through: (a) relative position of the magnets, superconductor, and magnetic field during field trapping; (b) relative position of the vertical external guide fields and trapped field during PNI measurement; (c) relative position of the horizontal external guide fields.
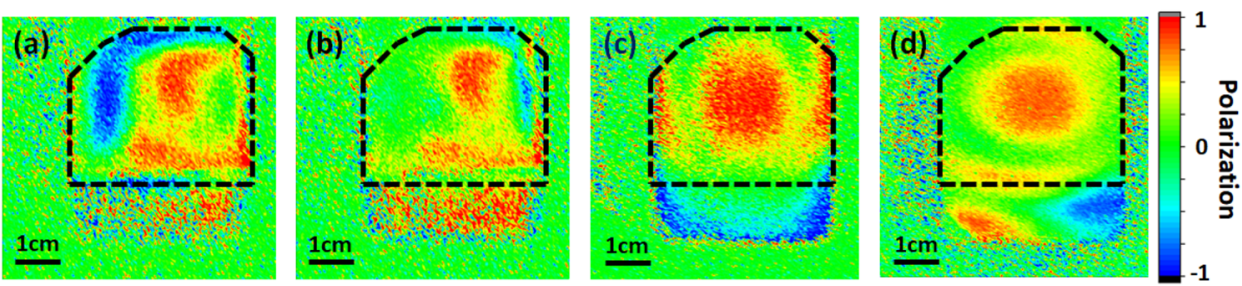

FIG. 9. PNI measurement result with the FC trapped field orthogonal to the sample surface using the setup described in Fig. 8. The results of the magnetic field trapped within the YBCO block are shown in (a) and (b), where the two electromagnetic fields are both horizontal [for (a)] or both vertical [for (b)]. The results of the magnetic field trapped within YBCO thin films are shown in (c) and (d) with the two electromagnets fields placed horizontally and vertically. 
flux at the surface of the superconductor. Based on this observation, it is possible to experimentally probe the magnitude of the trapped flux by varying the guide field magnitude. Unfortunately for our current setup, a controllable guide field that can reach 200 $\mathrm{G}$ is not yet available but will be developed in future experiments. Comparing results from the YBCO block and thin films, adiabatic neutron polarization transfer is observed in both cases near the center of the pattern. This result suggests that the neutron polarization transfer through the field trapped inside the YBCO block remains adiabatic.

\section{CONCLUSION}

Our experiments explored and demonstrated that wellcontrolled guide fields around the measured sample are essential to PNI measurements. This requirement is especially true for superconductors with the Meissner effect that distorts the magnetic field. For future measurement that quantitatively determines the internal magnetic field strength and distribution of a sample, instrument upgrade that enables neutron computed tomography (nCT) and sample rotation is needed. In addition, the current measurement is limited by its resolution of $350 \mu \mathrm{m}$, which can be further improved for investigation of flux pinning within the superconductor. From our observation, the trapped field is uniform up to our resolution limitation. Improving the resolution of PNI techniques to the size of type-II superconductor domains will provide more insight into quantum materials and is our major focus for future development.

\section{ACKNOWLEDGMENTS}

We would like to thank Dr. Fankang Li at ORNL for help in creating the model in Magnet ${ }^{(}$. This work was supported by the U.S. Department of Energy (DOE), Office of Science (OS), Basic Energy Sciences (BES), Materials Sciences and Engineering Division (sample design, fabrication, and physical property characterization), and by the Laboratory Directed Research and Development Program of Oak Ridge National Laboratory (ORNL), managed by UT-Battelle, LLC, for the U. S. DOE (PNR). The research at ORNL's High Flux Isotope Reactor was sponsored by the Scientific User Facilities Division, BES, U.S. DOE. Part of Tong's work was supported by the National Nature Science Foundation of China (No. 11875265) and by the Chinese Academy of Sciences.
This manuscript has been authored by UT-Battelle, LLC under Contract No. DE-AC05-00OR22725 with the U.S. Department of Energy. The United States Government retains and the publisher, by accepting the article for publication, acknowledges that the United States Government retains a non-exclusive, paid-up, irrevocable, worldwide license to publish or reproduce the published form of this manuscript, or allow others to do so, for United States Government purposes. The Department of Energy will provide public access to these results of federally sponsored research in accordance with the DOE Public Access Plan (http://energy.gov/downloads/doe-publicaccess-plan).

\section{REFERENCES}

${ }^{1}$ E. M. Forgan, S. J. Levett, P. G. Kealey, R. Cubitt, C. D. Dewhurst, and D. Fort, Phys. Rev. Lett. 88(16), 167003 (2002).

${ }^{2}$ G. Blatter, M. V. Feigelman, V. B. Geshkenbein, A. I. Larkin, and V. M. Vinokur, Rev. Mod. Phys. 66(4), 1125 (1994).

${ }^{3}$ N. Kardjilov, I. Manke, M. Strobl, A. Hilger, W. Treimer, M. Meissner, T. Krist, and J. Banhart, Nat. Phys. 4(5), 399 (2008).

${ }^{4}$ M. Dawson, I. Manke, N. Kardjilov, A. Hilger, M. Strobl, and J. Banhart, New J. Phys. 11, 043013 (2009).

${ }^{\mathbf{5}}$ W. Treimer, O. Ebrahimi, and N. Karakas, Appl. Phys. Lett. 101(16), 162603 (2012).

${ }^{6}$ W. Treimer, O. Ebrahimi, N. Karakas, and R. Prozorov, Phys. Rev. B 85(18), 184522 (2012).

${ }^{7}$ I. Manke, N. Kardjilov, A. Hilger, M. Strobl, M. Dawson, and J. Banhart, Nucl. Instrum. Methods A 605(1-2), 26 (2009).

${ }^{8}$ N. Kardjilov, A. Hilger, I. Manke, R. Woracek, and J. Banhart, J. Appl. Crystallogr. 49, 195 (2016)

${ }^{9}$ O. Schaerpf, Neutron Spin Echo (1980), p. 27.

${ }^{10}$ P. A. Seeger and L. L. Daemen, Nucl. Instrum. Methods A 457(1-2), 338 (2001).

${ }^{11}$ M. Sales, M. Strobl, T. Shinohara, A. Tremsin, L. T. Kuhn, W. R. B. Lionheart, N. M. Desai, A. B. Dahl, and S. Schmidt, Sci. Rep. 8(1), 2214 (2018).

${ }^{12}$ L. Landau, Nature 141, 688 (1938).

${ }^{13}$ C. P. Poole, R. Prozorov, H. A. Farach, and R. J. Creswick, in Superconductivity, 3rd ed., edited by C. P. Poole, R. Prozorov, H. A. Farach et al. (Elsevier, London, 2014), p. 183.

${ }^{14}$ C. Y. Jiang, X. Tong, D. R. Brown, S. Chi, A. D. Christianson, B. J. Kadron, J. L. Robertson, and B. L. Winn, Rev. Sci. Instrum. 85(7), 075112 (2014).

${ }^{15}$ I. Dhiman, R. Ziesche, T. H. Wang, H. Bilheux, L. Santodonato, X. Tong, C. Y. Jiang, I. Manke, W. Treimer, T. Chatterji, and N. Kardjilov, Rev. Sci. Instrum. 88(9), 095103 (2017)

${ }^{16}$ T. Wang, S. R. Parnell, W. A. Hamilton, F. Li, A. L. Washington, D. V. Baxter, and R. Pynn, Rev. Sci. Instrum. 87(3), 033901 (2016). 